\title{
Congress Calendar
}

\section{European Urology}

\subsubsection{0}

Stockholm

Sweden

14.6.-15.6.2000

Lipari, Eolian Islands

Italy

15.6.-17.6.2000

Lodz

Poland

25.6.-28.6.2000

Paris

France

31.8.-2.9. 2000

Malmö

Sweden

14.9.-16.9.2000

Interlaken

Switzerland
Public Health and Clinical Significance of Premalignant Alterations in the Genitourinary Tract

Who Consensus Conference

Urology 2000: State of the Art - Looking at the Future

XXXth Jubilee Scientific Congress of the Polish Association of Urology

5th International Consultation on Benign Prostatic Hyperplasia

1st International Consultation on Nosocomial Infections in Urology

Nordic Association for Andrology, Annual Meeting 2000

56ème Assemblée de la Société Suisse d'Urologie
Information: Prof. Lennhart Andersson, WHO

Collaborating Center for Urologic Tumors,

Karolinska Hospital, SE-171 76 Stockholm (Sweden), Fax +468326113

Information: Prof. Carlo Aragona, Unità Operativa di Urologia, Azienda Ospedaliera Papardo, Contrada Parpardo, I-98158 Messina (Italy), Tel. +39090 3992491/+390903992281,

Fax +39090 3992284, E-Mail carlo.aragona@mail.inet.it

Information: Prof. Leszek Jeromin, Chairman of the Organizing Committee, Urology Clinic, Pabianicka 62, P-93 513 Lodz (Poland) Tel. +48 (42) 68440 57, Fax +48 (42) 6849850 E-Mail: recepcja@uro.congress2000.lodz.pl http://www.uro.congress2000.lodz.pl/

Information: Dr. Saad Khoury,

Clinique Urologique (Prof. Richard), Hôpital de la Pitié, 83, bd de l'Hôpital F-75634 Paris Cedex (France),

Tel. +331421771 20, Fax +33142177122, E-Mail: s.khoury@wanadoo.fr

Information: Jörgen Oldbring, Department of Urology, Malmö University Hospital, SE-205 02, Malmö (Sweden) Tel. +46 40 333752, Fax +46 40337049 ,

E-Mail: jorgen.oldbring@kir.mas.lu.se

Information: Prof. H.-J. Leisinger, Service d'Urologie, CHUV BH-10, CH-1011 Lausanne (Switzerland)

Tél. +41 2131429 81, Fax +41 2131429 82,

E-Mail: Hans-Jurg.Leisinger@ chuv.hospvd.ch 


\section{European Urology}

\section{9.-17.9.2000 \\ Cleveland Mariott}

Ohio, (USA)

5.10.-6.10.2000

Munich

Germany

\subsection{0.-7.10.2000}

Istanbul

Turkey

6.10.-7.10.2000

Varese

Italy

7.10.-9.10.2000
Kos Island
Greece

8.10.-10.10.2000

Parma

Italy

\subsection{0.-12.10.2000 \\ Izmir}

Turkey

19.10.-21.10.2000

Marburg

Germany

26.10.-29.10.2000

\section{Bologna}

Italy

23.11.-30.11.2000
Erice

Italy 2nd Annual Fall Research Meeting of the Society for the Study of Impotence (SSI)

28th Munich Endourological Symposium with Nursing Staff Seminar

15th ESUOE Congress of the European Society for Urological Oncology and Endocrinology

European Society of Pediatric Urology (ESPU)

Annual Course on Hypospadias

2nd International Congress of the History of Urology

Urology through the Ages and the Role of Hippocratic Medicine (Past, Present and Future)

Xth National Congress S.I.Ur.O

(Italian Society of Oncological Urology)

Promoter: Dr. Domenico Potenzoni

16th National Congress of the Turkish Urological

Association (TUA)

4th International Symposium on Uro-Oncology

'Advances in Diagnosis and Treatment of Prostate Cancer'

Including Live Surgery

XXIVth National Congress SIUD

(Italian Urodynamic Society)

Promoter: Prof. Giuseppe Martorana

9th Course of International School of Urology and

Nephrology of Ettore Majorana
Information: Secretariat SSI, 1111 North Plaza Drive, Suite 550, Schaumburg, IL 60173 (USA) Tel. +1 (847) 517 7225, Fax +1 (847) 5177229 E-Mail: ssi@wjweiser.com

Information: Dr. R. Paul, Department of Urology, Technische Universität München, Klinkum rechts der Isar, Ismaningerstrasse 22, D-81675 München (Germany), Tel. +49894140 2507, Fax +498941402585, E-Mail: mriu@1rz.tu-muenchen.de,

http://www.med.tu-muenchen.de/uro/endo.html

Information: Prof. Dr. Atif Akdas, Assoc. Prof. Dr. Levent Turkery, Department of Urology, Marmara University, School of Medicine, Tophanelioglu cad. No. 13-15, Altunizade, TR-81190 Istanbul (Turkey),

Tel. +90 216325 2052, Fax +90 2163258679 . E-Mail: urology@marun.edu.tr

Information: Dr. G.A. Manzoni, Section of Pediatric Urology and Department of Urology, Ospedale di Circolo, I-21100 Varese (Italy) Tel. +390332 260207, Fax +390332520620, E-Mail: urolva@ats.it

Information: Erasmus Conference Center, 227 Kifissias Ave., 14561 Kifissia, Athens (Greece), Tel + +30 $16125022-3$, Fax +3016125021, E-Mail: erasmus@netit.gr

Information: Secretariat euro conventions Via Torricella, 14, I-29100 Piacenza (Italy) Tel. +39 (0) 5233357 32, Fax +39 (0) 523334997 E-Mail: info@euroconventions.it

Information: Assoc. Prof. Dr. Mete Çek, Assoc. Prof. Dr. Tarik Esen, Turkish Urological Association, Nurettin Öktem Sok. 18/2, TR-80260 Sisli-Istanbul (Turkey) Fax +90 212233 9804; E-Mail: info@uroturk.org

Information: Priv. Doz. Dr. A. Heidenreich, Department of Urology, Philips-Universität Marburg, Baldingerstrasse, D-35043 Marburg (Germany) Tel. +496421286 6239, Fax +496421286 5590,

E-Mail: heidenre@post.med.uni-marburg.de

Information: Secretariat euro conventions Via Torricella, 14, I-29100 Piacenza (Italy) Tel. +39 (0) 5233357 32, Fax +39 (0) 523334997 E-Mail: info@euroconventions.it

Information: Prof. Michele Pavone-Macaluso, Institute of Urology, University Policlinic Hospital, Via del Vespro, 129, I-90127 Palermo (Italy), Tel. +39 $091655240 / 4 / 5$, Fax +390916552434, E-Mail: michpav@tin.it 